\title{
reviews
}

columns



\section{A Multidisciplinary Handbook of Child and Adolescent Mental Health for Front-line Professionals}

Nisha Dogra, Andrew Parkin, Fiona Gale and Clay Frake

Jessica Kingsley publishers. 2002. 303 pp. ISBN: 1-85392-929-7

Training professionals from different theoretical backgrounds (social workers, nurses, educational psychologists) and experiences (class teachers, special education needs coordinators, learning support mentors, minibus escorts etc.) is a considerable challenge. A multidisciplinary handbook of child and adolescent mental health for front-line professionals was therefore very welcome.

The book is divided into seven parts covering most of the topics that are pertinent to child and adolescent mental health services. These are defining and meeting the mental health needs of children and young people, child and family development, factors that influence the mental health of young people, specific mental health problems of childhood and adolescence, treatment and management strategies, medico-legal aspects of child mental health, exercise and case study solutions. It does not attempt to describe in detail any of the topics, but provides enough information for the tier 1 professional in an easily accessible format. The authors come from a wide, multidisciplinary background.

The format uses lists of salient features, bullet points and boxes, making for an easy read. I particularly liked the exercises after each chapter in the first part and the case studies at the end of other chapters. The answers to all these exercises are available in Chapter 7.
The chapter on major mental health disorders is concise and well written with just enough information, as is the chapter on attention-deficit hyperactivity disorder.

The chapter on behaviour problems was disappointing. Behavioural problems in child and adolescent mental health services can be due to many factors, ranging from developmental disorders like autistic spectrum disorder and learning disability to a social/environmental reason, although in most cases it is due to multiple aetiological factors. The authors discuss presentation and risk factors, but do not mention assessment. Under management of this condition, I would have expected an acknowledgement that it differs depending on the varying aetiological factors and that getting the right formulation is crucial for the success of treatment. It reminded me of the account of a parent who I now invite to take part in training sessions for child and adolescent mental health service tier $2 / 3$ clinicians. This parent had been advised about the usual behavioural strategies by clinicians both in tier 1 and in tiers 2/3, but they had failed to consider a biopsycho-social approach in their assessment. The child later turned out to have autism. The mother was left with the feeling that she was useless and that her child's difficult behaviour was due to her inability to parent, so she did not engage in treatment. Though the book does not attempt to be a treatise for clinicians in this area and it is aimed at front-line professionals, I think it is equally important for them not to embark on treatment without an assessment that could potentially lead to the 'persecution' of parents when behavioural advice has not worked. The other criticism I have is that although parenting programmes are described in detail in Chapter 14, this is not specifically mentioned as a treatment option for behavioural problems, in spite of the good clinical evidence for its effectiveness.

In spite of these minor quibbles, I would recommend this book as a useful addition to primary care libraries where it could be accessed by tier 1 professionals such as teachers, health visitors and school nurses.

Latha Hackett, Consultant in Child and Adolescent Psychiatry, TheWinnicott Centre, 195-197 Hathersage Road, Manchester M13 0JE

\section{Family Focused Grief Therapy. A Model of Family- centred Care During Palliative Care and Bereavement}

David W Kissane, Sidney Bloch (eds). Open University Press, 2002

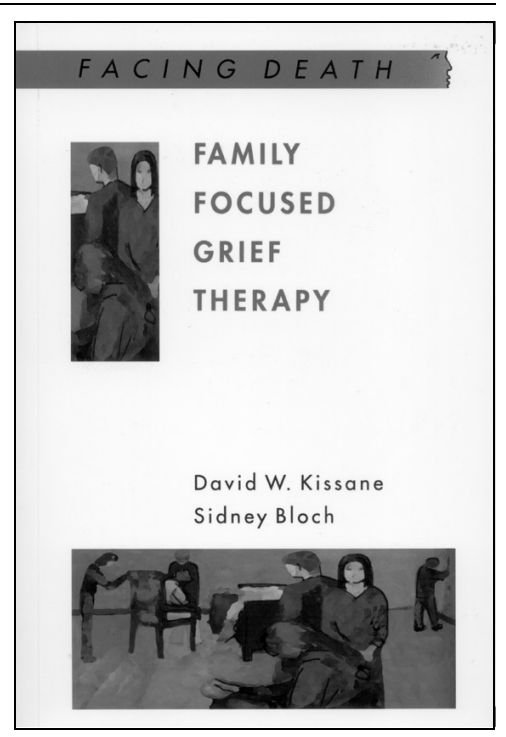

272 pp. f22.50 pb, f65.00 hb. ISBN: 0-335-203493

Death is not new; carefully conducted and well-presented research is a much less familiar achievement. You will not regret reading this book. The core of the book is the use of family focused grief therapy in a randomised, controlled trial extending over a 5 -year period and involving 81 families. That project, the Melbourne Family Grief Studies, represents a tour de force. For those who have an appetite for figures and statistics, the authors have provided an appendix that contain data from their project.

Over nine chapters, Kissane and Bloch introduce the reader to the systemic family approach to the management of grief (in the setting of palliative care), the rationale to family focused grief therapy, their research methods (including The Family Relationships Index), and the possible applications of the therapy, as well as the challenges that might face those who use their model of intervention. They demonstrate persuasively that this time-limited therapy identifies the family as the unit of care and that it can be used in different socio-cultural and clinical settings. It is amenable to empirical evaluation, and it is securely wedded to theoretical constructs, particularly those associated with attachment theory. This is not a sterile text. The authors reify their theoretical principles and concepts by presenting relevant clinical cases, some of which are very complex. I particularly liked their willingness to describe frankly how the cases had been managed, whatever the outcome.

While the authors articulate how to use family focused grief therapy, they show no sympathy for those who seek a 'therapy by numbers' approach. The family focused grief therapy requires training and 
supervision, as well as an understanding of family dynamics and a willingness to deal with families sensitively and flexibly.

Just when you think you have grasped the essentials to family focused grief therapy, you are then challenged with the penultimate chapter, which is dedicated to ethical issues. This is not a litany of pious prescriptions. It is a thought-provoking chapter of value to any practitioner, whatever their theoretical persuasion. The preeminent injunction is 'First do no harm'.

In conclusion, this book represents a genuine contribution to the repertoire of methods for helping families prior to and subsequent to the demise of a loved one It is well written in an unpretentious and frank style. The authors' wealth of clinical experience is evident in their writing, as is their ability to blend a scholarly and scientific approach with a compassionate and flexible clinical one.

David A Alexander Professor of Mental Health Medical School, University of Aberdeen, Director, Aberdeen Centre forTrauma Research

\section{miscellany}

\section{Mbarara University Teaching Hospital, Uganda}

A great opportunity exists for a psychiatrist to work in a new medical school at Mbarara University Teaching Hospital,
Uganda, and to help build up the academic and community psychiatry. The post would best suit a retired psychiatrist, a sabbatical holder or an enthusiastic specialist registrar. A local salary and free accommodation will be offered. Further enquiries should be sent to Professor Eldryd Parry, The Tropical Health and Education Trust, 24 Eversholt Street, London NW1 1AD (tel: 0207679 8127; fax: 0207679 8190; e-mail: epthet1@aol.com).

\section{forthcoming events}

Applications are invited for Semester One of the MRCPsych Course run by University College London. Part I takes place on Monday afternoons commencing 1 September 2003 and Part II takes place on Tuesday afternoons commencing 2 September 2003. The course has been updated in keeping with the Royal College of Psychiatrists' syllabus, and includes mock exams, theme specific revision sessions and patient perspectives. For further information and an application form, please contact Lydia M. Clinton, the Course Administrator (tel: 0207679 9475; e-mail: mrcpsych@ucl.ac.uk).

The 1st Edinburgh MRCPsych part II Critical Appraisal Exam Course will be held at Pollock Halls, Edinburgh, on the weekend of 6-7 September 2003. The course includes plenary lectures, guided critical appraisal, troubleshooting and how to approach the exam sessions, and a mock exam (with model answers supplied afterwards); organised and presented by the authors of Critical Appraisal for Psychiatry. Overnight on-site inexpensive accommodation is available. For further details and application forms, please contact Ms Norma Brearley, Kennedy Tower, Royal Edinburgh Hospital, Morningside Place, Edinburgh EH10 5HF or visit the departmental website at http:// www.pst.ed.ac.uk/index.shtml.

Mole Conferences would like to announce the following course:

An introduction to cognitivebehavioural therapy. This 2-day basic introductory workshop will take place on 10-11 November 2003 in central London. The event is aimed at those with little or no understanding or experience of this way of working, and aims to provide those attending with practical skills that can be used safely with clients following completion of the course. The course includes an examination paper and is a generic course, appropriate for staff working in a diversity of settings. The course will involve basic theory, the therapeutic alliance, assessment and case information, structuring sessions, using homework, therapeutic assignments and designing behavioural experiments, thinking skills and effective challenging of unhelpful thinking styles. For further information please contact Mole Conferences, 26 Church Road, Portslade, Brighton BN41 1LA (tel: 01273 242634; fax: 01273 235095; e-mail: enquiries@mole-conferences.com; website and online booking: www.mol-conferences com)

Clinical and research refresher course on psychological science and mental health issues is the title of a course being held on 27--28 November 2003 at the Institute of Psychiatry. This course, organised by the Department of Psychology, will provide participants with a comprehensive update on empirically grounded developments in the psychological understanding and management of a range of problems. In addition, some presentations will present the latest thinking on key psychological topics which are becoming increasingly influential in the development of psychological neuroscience. There will be a range of topics covered in the two days including the following: Psychosis: understanding and treatment, Bipolar Disorder, Mindful- ness, Forensic, Genetics, Anxiety Disorders, Post Traumatic Stress Disorder, Neuropsychology, Virtual Reality, Children, Families and Cognitive-Behavioural Therapy, Health Anxiety and Substance Misuse. For further information, please contact Lesley Anderson, Department of Psychology, Box P077, Institute of Psychiatry, De Crespigny Park, London SE5 8AF (tel: 0207848 5038; e-mail: l.anderson@iop.kcl.ac.uk).

The International Conference on Schizophrenia (ICONS) organised by the Schizophrenia Research Foundation (SCARF) India, a World Health Organization Collaborating Centre for Mental Health Research \& Training, and co-sponsored by the World Psychiatric Association will take place on 29 January-2 February 2004 at Chennai (formerly Madras), India. This meeting will showcase the best of schizophrenia research from around the world. There will be a one-day workshop by Dr Judith Jaeger on Cognition in Schizophrenia (2 February 2004) with limited registrations. Themes will include: Epidemiology, Phenomenology, Course \& Outcome, Biology, Neuropsychology, Drug Treatment, Disability \& Rehabilitation, and Culture \& Psychiatry. Key Note Speakers will be Professor Ahamed Okasha, President, World Psychiatric Association and Dr Benedetto Saraceno, Director Mental Health Division, World Health Organization. The deadline for submission of abstracts is September 2003. For further details, please contact Dr R. Thara, Director, SCARF (e-mail: info@icons-scarf.org or scarf@vsnl.com). Alternatively, visit http://www.iconsscarf.org 